\title{
Re-reading ACT, BCG, and Low COVID-19 in Africa
}

\author{
Jia Bainga Kangbai ${ }^{1,2,3}$ - Lawrence Sao Babawo $^{3,4} \cdot$ Daniel Kaitibi $^{5} \cdot$ Anthony A. Sandi $^{6}$. \\ Angela Magdalene George ${ }^{4} \cdot$ Foday Sahr ${ }^{3,7,8}$
}

Accepted: 9 December 2020 / Published online: 12 January 2021

(C) The Author(s), under exclusive licence to Springer Nature Switzerland AG part of Springer Nature 2021

\begin{abstract}
October 11, 2020, marks the seventh month since the World Health Organization (WHO) officially declared COVID-19 a pandemic. Unlike other coronavirus diseases, there is a geographically disproportionate distribution of the incidence of COVID-19 cases around the world. We observed a significantly high COVID-19 cases and deaths in countries and territories with no or very small number of malaria cases or no or low national TB cases in 2018 . We speculate that the high incidence of COVID-19 cases and deaths in countries less affected by malaria is partly due to overexposure to malaria which led to the regular use of the artemisinin anti-malaria drugs as well as the regular use of bacillus Calmette-Guérin (BCG) vaccine for TB prevention. The vaccine produced an almost life-long immunity to TB and meningitis to its recipients. We are thus calling for a COVID-19 containment and clinical management protocol that will incorporate the use of the anti-malaria ACT drug cocktail and BCG vaccine on compassionate ground.
\end{abstract}

Keywords COVID-19 $\cdot$ Pandemic $\cdot$ Malaria $\cdot$ Tuberculosis $\cdot$ Coronavirus $\cdot$ Artemisinin $\cdot$ Incidence

\section{Dear Editor}

October 11, 2020, marks the seventh month since the World Health Organization (WHO) officially declared COVID-19 a pandemic. Unlike other coronavirus diseases, there is a geographically disproportionate distribution of the incidence of COVID-19 cases around the world. As of October 12, 2020, COVID-19 is affecting 214 countries and territories with Qatar (45,655 per million), Aruba (39,263 per million), Andora (38,745 per million), French Guyana (33,842 per

This article is part of the Topical Collection on Covid-19

Jia Bainga Kangbai

Jia.Kangbai@lrz.uni-muenchen.de

Lawrence Sao Babawo

sababawo@njala.edu.sl

Daniel Kaitibi

dkiatibie@njala.edu.sl

Anthony A. Sandi

aasandi@njala.edu.sl

Angela Magdalene George

ageorge@njala.edu.s1

Foday Sahr

Fsahr@usl.sl million), Israel (31,968 per million), Kuwait (26,093 per million), Peru (25,715 per million), Chile (25,200 per million), USA (24,243 per million), and Brazil (23,961 per million) [1] recording the highest cases (Fig. 1).

The COVID-19 pandemic has severely affected the management of other diseases such as malaria and tuberculosis (TB). Globally, there were more than 200 million malaria cases culminating into about 500,000 deaths in 2018; India and 19 Africa countries accounted for about 85\% [2]. Additionally, there were 10 million TB cases and about 1.5

Center for International Health, University of Munich (LMU), Munich, Germany

2 Department of Environmental Health Sciences, Njala University, Freetown, Sierra Leone

3 The National COVID Emergency Response Centre (NACOVERC), Freetown, Sierra Leone

4 Department of Nursing, Njala University, Freetown, Sierra Leone

5 Njala University, Freetown, Sierra Leone

6 Ministry of Health and Sanitation, Freetown, Sierra Leone

7 Department of Microbiology, College of Medicine and Allied Health Sciences, University of Sierra Leone, Freetown, Sierra Leone

8 The 34 Military Hospital, Wilberforce, Freetown, Sierra Leone 
Fig. 1 Distribution of COVID-19 cases and deaths by countries as of October 12, 2020

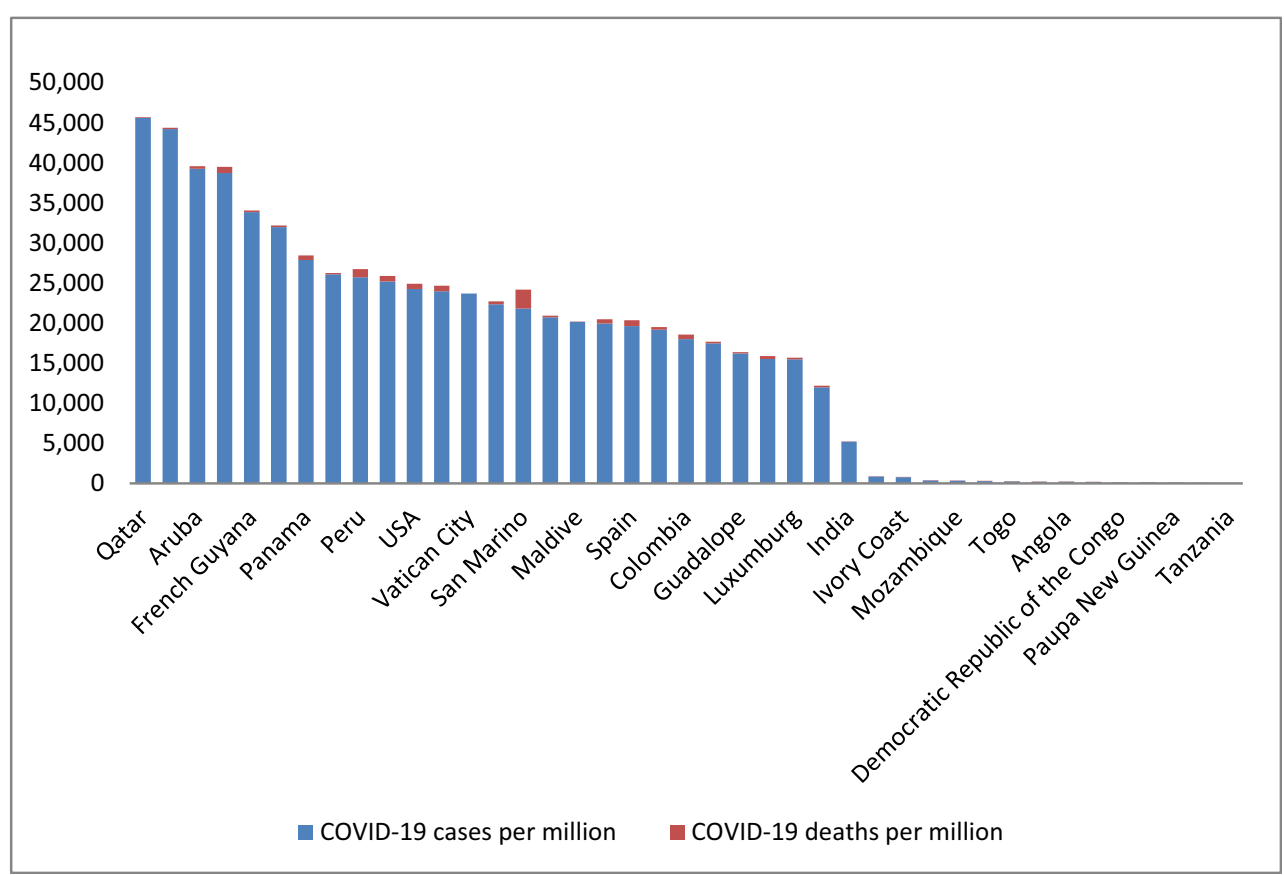

million TB-related deaths globally in 2018, a reduction of 2 and $5 \%$, respectively, from 2017. Two-thirds of the global TB cases were in eight countries: India $(27 \%)$, China $(9 \%)$, Indonesia (8\%), Philippines (6\%), Nigeria (4\%), Bangladesh (4\%), and South Africa (3\%) [2].

Of those countries and territories with > 11,000 COVID-19 cases as of October 12, 2020, only Andora (399 malaria cases per million) and Brazil (320 malaria cases per million) recorded comparatively high incidence of malaria in 2018 [2]. We observed a significantly high COVID-19 cases and deaths in countries and territories with no or very small number of malaria cases. For instance, Qatar (45,655 COVID-19 cases per million), Bahrain (44,214 COVID-19 cases per million), Aruba (39,263 COVID-19 cases per million), French Guyana (33,842 COVID-19 cases per million), Israel (31,968 COVID-19 cases per million), Panama $(27,874$ COVID-19 cases per million), Kuwait (26,093 COVID-19 cases per million), Peru (25,715 COVID-19 cases per million), and Chile (25,200 COVID-19 cases per million) reported 0.00001 malaria cases per million, 0.00001 malaria cases per million, 4 malaria cases per million, 0.00001 malaria cases per million, 0.00001 malaria cases per million, 0.0002 malaria cases per million, 6 malaria case per million, 0.00001 malaria cases per million, and 0.00001 malaria cases per million, respectively [2] (Fig. 2).

From Fig. 2, countries with high COVID-19 cases and deaths recorded no or very little malaria cases in 2018, while countries with significantly high malaria cases recorded no or very little COVID-19 cases or deaths.

We also observed a significantly high COVID-19 cases and deaths in countries and territories with no or low national
TB cases in 2018. For instance, as of October 12, 2020, and with the exception of Peru (with 25,715 COVID-19 cases and 1008 COVID-19 deaths per million and 123 TB cases per 100,000), all countries with $>11,000$ COVID-19 cases recorded $<100$ TB cases per 100,000 population at risk. Additionally, countries with high TB burden in 2018 recorded less COVID-19 mortality rates than those with low TB burden. For instance, India (5184 COVID-19 cases and 79 deaths per million and 199 TB cases per 100,000), Zambia (839 COVID-19 cases and 19 deaths per million and 346 TB cases per 100,000), Ivory Coast (759 COVID-19 cases and 5 deaths per million and 195 TB cases per 100,000), Mozambique (320 COVID-19 cases and 2 deaths per million and 551 TB cases per 100,000), Nigeria (291 COVID-19 cases and 5 COVID19 deaths per million and 219 TB cases per 100,000), Uganda (214 COVID-19 cases and 2 deaths per million and 200 TB cases per 100,000), Angola (196 COVID-19 cases and 7 deaths per million and 355 TB cases per 100,000), Democratic Republic of Congo (120 COVID-19 cases and 3 deaths per million and 322 TB cases per 100,000), Papua New Guinea (63 COVID-19 cases and 0.8 deaths per million and 388 TB cases per 100,000), and Tanzania (8 COVID-19 cases and 0.3 deaths per million and 253 TB cases per 100,000) recorded low COVID-19 case fatalities and > 190 TB cases per 100,000 of population at risk [3] (Fig. 3).

From Fig. 3, countries with considerably high TB burden recorded very low COVID-19 case fatalities.

We used Poisson regression to assess whether the numbers of malaria and TB cases reported in 2018 are predictors for COVID-19 cases. Holding the number of TB cases in the model constant, as the number of malarial cases increases by 
Fig. 2 Distribution of COVID-19 cases and deaths by countries as of October 12, 2020, alongside with the number of reported malaria cases for 2018

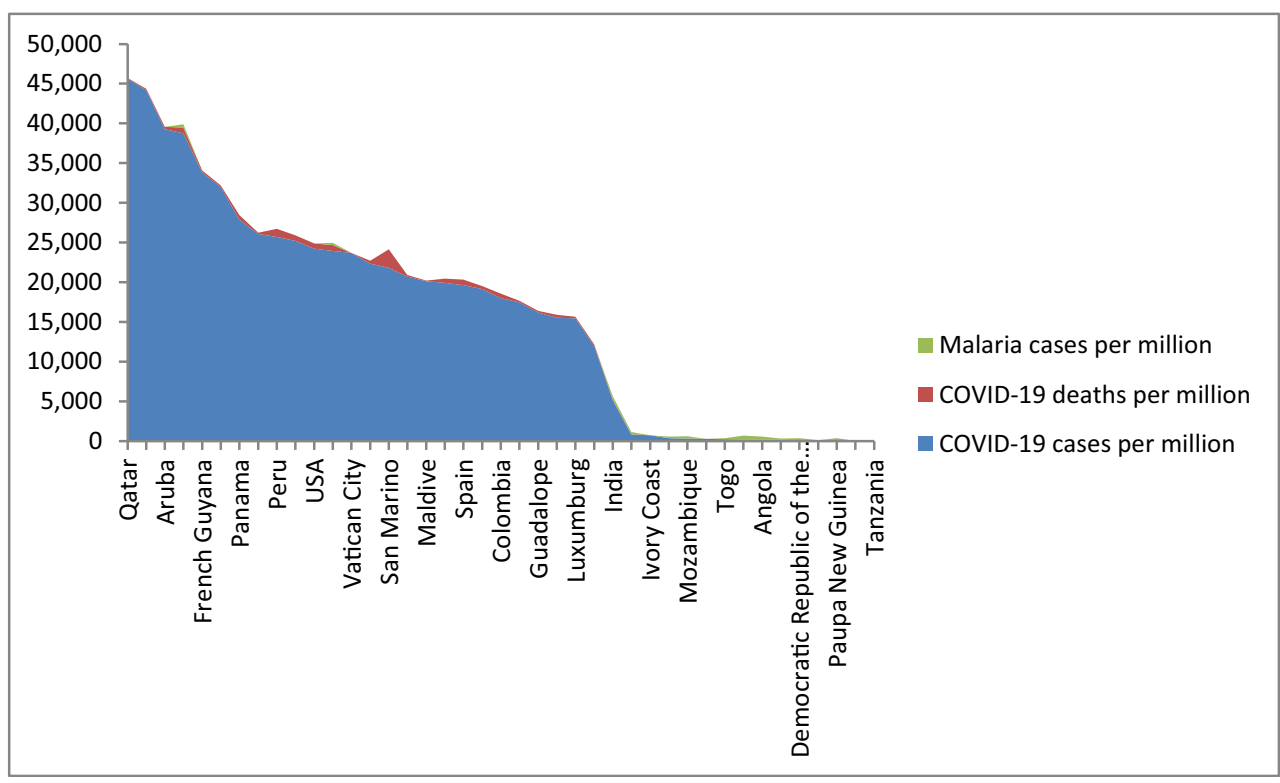

1 (per million at risk), the COVID-19 incidence rate decrease by 10.00 (per million at risk). Additionally, holding the number of malaria cases in the model constant, as the TB cases increase by 1 (per million at risk), the COVID-19 incidence rate decreases by 9.90 (per million at risk). The AUC for the ROC curve analysis was $89.5 \%$, which shows that COVID-19 incidence rate was a good classifier for malaria-free countries or those countries with limited malarial infections (4 or less malaria cases per 1 million at risk).

We speculate that the low incidence of COVID-19 cases and deaths in countries highly affected by malaria is partly due to overexposure to malaria which led to the regular use of the artemisinin anti-malaria drugs, as well as the use of BCG vaccination for TB. Our speculation is based on the fact that several African countries that are endemic to malaria continue to record very low COVID$19[4,5]$ incidence rates compared to non-Africa countries that are not endemic to malaria. We do not go along with the idea that the low COVID-19 incident rates among black people may be due to genetic susceptibility since COVID-19 severity among African-Americans in the USA is very high. Additionally, we are also speculating that the low COVID-19 incidence rates recorded by malaria endemic countries may be due to anti-malaria immunity produced by antibodies ( $\mathrm{IgG}$ ) following exposure to plasmodium-specific antigens. Overexposure to a particular pathogen ensures a huge stockpile of antigen-specific antibodies for that pathogen.
Fig. 3 Distribution of COVID-19 deaths by countries as of October 12,2020 , alongside with number of reported TB cases in 2018

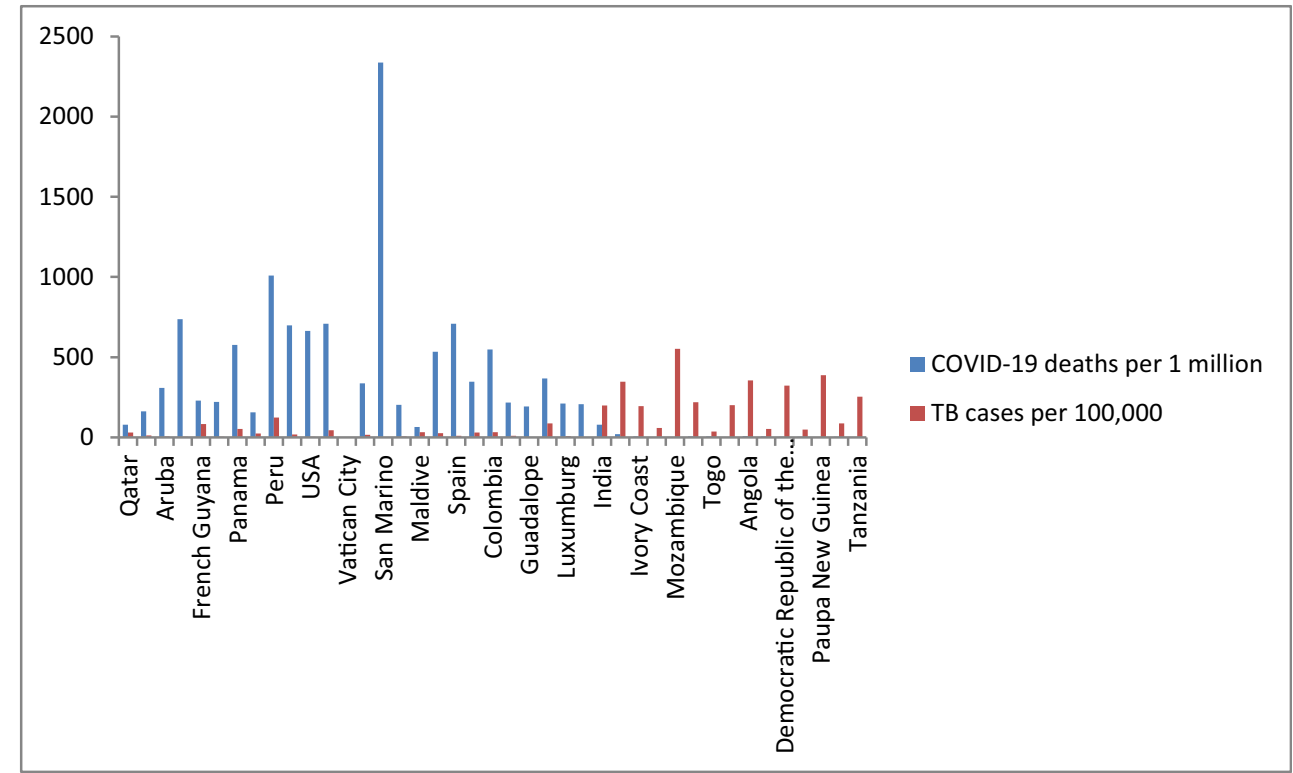


One study reported that people who have antibodies to different pathogens are known to be protected against COVID-19 [6]. The IgG that is produced by plasmodiumspecific antigens can recognize the different COVID-19 glycoproteins (GPs) including membrane GPs such as spike GPs and GPs that have acetyl esterase and hemagglutination properties [7], thereby either providing protection against COVID19 or producing a much milder infection [8]. Another possible explanation of the low transmission of COVID-19 in countries endemic with malaria is the wide use of the anti-malaria artemisinin drug. Unlike chloroquine that was withdrawn in the late 1990s due to growing resistance to it by the plasmodium pathogen [9-12], most $(n=65 / 67,97 \%)$ malaria endemic countries including 41 Africa countries adopted artemisinin combine terapy (ACT) as their first line of treatment for uncomplicated malaria in 2000 [13]. One study has demonstrated in vitro the clinical effectiveness of artemisinin in the treatment of COVID-19 [14].

The low COVID-19 incidence rates in countries with high TB cases as reported in 2018 may be associated with the wide use of the bacillus Calmette-Guérin (BCG) vaccine which offers protection against both TB and meningitis. BCG vaccine is most commonly administered to persons below 35 years and who are at high risk of becoming infected with $\mathrm{TB}$ in their life time. The vaccine produced an almost life-long immunity to TB and meningitis recipients. However, there have been opposing views whether BCG can offer protection from COVID-19 infection [15-17].

We are also aware that Africa's low COVID-19 cases may have been due to the limited COVID-19 testing as well as the limited COVID-19 testing data coming from the continent. With such limitations, it is still difficult to conclude whether the spread of COVID-19 in Africa is minimal. We also agree with those who are claiming that Africa population may have been spared the full brunt of the pandemic because of the continent's high youthful population, low prevalence of cardiovascular diseases, limited international connectivity, and the continent's strong public health systems. The Africa continent with 54 countries, different infrastructures, and diverse health policies is yet characterized by having a youthful population. One paper pointed out that having a large youthful population may mean many infected COVID-19 patients not displaying symptoms thereby placing many other people at risk than would symptomatic individuals [18]. In one Chinese study, only $1 \%$ of the 44,672 COVID-19 cases were below 10 years [19], while another study reported that children just like adult got infected and that community transmission of COVID-19 may be link to pediatric COVID-19 cases [20].

Comparatively to Europe and North America, Africa continent has lower prevalence of noncommunicable diseases (NCD) such as cardiovascular diseases, cancer, and diabetes, all of which places COVID-19 patients at high mortality risk. According to the World Health Organization, 17.9 million people died annually from cardiovascular diseases, 9.0 million died from cancers, and 3.9 million people died annually from respiratory diseases, while 1.6 million people died from diabetes [21]. There has been a rising trend in the prevalence of NCD in Africa, but the continent is still trailing Europe and North America in terms of NCD morbidity and mortality; the burden of disease in Africa is still dominated by infectious diseases [22].

We are thus calling for a COVID-19 containment and clinical management protocol that will incorporate the use of the anti-malaria ACT drug cocktail and BCG vaccine on compassionate ground. Additionally, future studies regarding the clinical effectiveness of ACT and BCG vaccine on COVID-19 infection will be desirable.

\section{Compliance with Ethical Standards}

Competing Interests All authors declare they have no competing interest.

\section{References}

1. Worldometer.info. (https://www.worldometers.info/coronavirus/. [accessed on October 12, 2020]; Dover, Delaware, USA.

2. The "World Malaria report 2019" report at a glance. World Health Organisation. [accessed on October 12, 2020].

3. Global Tuberculosis Report 2018. World Health Organisation. [accessed on October 12, 2020].

4. Ahmed AE. Incidence of coronavirus disease (COVID-19) and countries affected by malarial infections. Travel Med Infect Dis. 2020;37:101693. https://doi.org/10.1016/j.tmaid.2020.101693.

5. Muneer A, et al. Comparative analysis revealed reduced spread of COVID-19 in malaria endemic countries. medRxiv preprint. https:// doi.org/10.1101/2020.05.11.20097923

6. Napoli PE, Nioi M, d'Aloja E, Fossarello M. Safety recommendations and medical liability in ocular surgery during the COVID-19 pandemic: an unsolved dilemma. J Clin Med; 9:E1403.d.

7. Parodi A, Cozzani E. Coronavirus disease 2019 (COVID 19) and malaria have anti glycoprotein antibodies a role? Med Hypotheses. 2020;143:110036

8. Gomes LR, Martins YC, Ferreira-da-Cruz MF, Daniel-Ribeiro CT. Autoimmunity, phospholipid-reacting antibodies and malaria immunity. Lupus. 2014;23:1295-8.

9. Kublin JG, Cortese JF, Njunju EM, Mukadam RA, Wirima JJ, Kazembe PN, et al. Reemergence of chloroquine-sensitive Plasmodium falciparum malaria after cessation of chloroquine use in Malawi. J Infect Dis. 2003;187:1870-5. https://doi.org/10.1086/ 375419.

10. Laufer MK, Thesing PC, Eddington ND, Masonga R, Dzinjalamala FK, Takala SL, et al. Return of chloroquine antimalarial efficacy in Malawi. N Engl J Med. 2006;355:1959-66. https://doi.org/10. 1056/NEJMoa062032.

11. Eriksen J, Nsimba SE, Minzi OM, Sanga AJ, Petzold M, Gustafsson LL, et al. Adoption of the new antimalarial drug policy in Tanzania-a cross-sectional study in the community. Tropical Med Int Health. 2005;10:1038-46. https://doi.org/10.1111/j.13653156.2005.01486.x. 
12. Kamya MR, Bakyaita NN, Talisuna AO, Were WM, Staedke SG. Increasing antimalarial drug resistance in Uganda and revision of the national drug policy. Tropical Med Int Health. 2002;7:1031-41. https://doi.org/10.1046/j.1365-3156.2002.00974.x.

13. Bosman A, Mendis KN. A major transition in malaria treatment: the adoption and deployment of artemisinin-based combination therapies. Supplement to volume 77(6). American journal of tropical medicine and hygiene. Northbrook (IL): American Society of Tropical Medicine and Hygiene; 2007.

14. Gendrot M, Duflot I, Boxberger M, Delandre O, Jardot P, le Bideau $\mathrm{M}$, et al. Antimalarial artemisinin-based combination therapies (ACT) and COVID-19 in Africa: in vitro inhibition of SARS$\mathrm{CoV}-2$ replication by mefloquine-artesunate. Int $\mathrm{J}$ Infect Dis. 2020;99:437-40. https://doi.org/10.1016/j.ijid.2020.08.032.

15. Cecilia S, Arlehamn L, Alessandro Sette A, Peters B. Lack of evidence for BCG vaccine protection from severe COVID-19. PNAS. 2020;117(41):25203-4; first published September 29, 2020. https:// doi.org/10.1073/pnas.2016733117.

16. Escobar LE, Molina-Cruz A, Barillas-Mury C. BCG vaccine protection from severe coronavirus disease 2019 (COVID-19). PNAS. 2020;117(30):17720-6; first published July 9, 2020; updated October 12, 2020. https://doi.org/10.1073/pnas.2008410117.
17. Mohapatra PR, et al. BCG vaccination induced protection from COVID-19. Indian J Tuberc. 2020. https://doi.org/10.1016/j.ijtb. 2020.08.004.

18. Lee P-I, Hu Y-L, Chen P-Y, et al. Are children less susceptible to COVID-19? J Microbiol Immunol Infect. 2020;53:371-2.

19. Wu Z, McGoogan JM. Characteristics of and important lessons from the coronavirus disease 2019 (COVID-19) outbreak in China: summary of a report of 72314 cases from the Chinese Center for Disease Control and Prevention. JAMA. Published online February 24, 2020. https://doi.org/10.1001/jama.2020.2648.

20. $\mathrm{Bi} \mathrm{Q}$, et al. Epidemiology and transmission of COVID-19 in Shenzhen China: Analysis of 391 cases and 1,286 of their close contacts. https://doi.org/10.1101/2020.03.03.20028423

21. WHO. Noncommunicable diseases and mental health. Published September 2018. https:/www.who.int/nmh/publications/ncdprofiles-2018/en/

22. Gouda HN, Charlson F, Sorsdahl K, Ahmadzada S, Ferrari AJ, Erskine H, et al. Burden of non-communicable diseases in subSaharan Africa, 1990-2017: results from the global burden of disease study 2017. Lancet Glob Health. 2019;7:e1375-87.

Publisher's Note Springer Nature remains neutral with regard to jurisdictional claims in published maps and institutional affiliations. 